\title{
NEGLECTED TROPICAL DISEASES IN GHANA: CURRENT STATE AND THE WAY FORWARD
}

\section{Paul Armah Ndabiah, Dmitry Kicha}

\author{
People's Friendship University of Russia (RUDN), Moscow, Russia \\ Paularmah60@yahoo.com
}

\begin{abstract}
Health policies are correlated with the development of the society both nationally and locally. On the other hand, the allocation of resources for health services is a priority in regions with a lower degree of local development, where the health of the population is higher because of the more difficult access to health services, the lack of information and the postponement of the decision because of the lack of personal health care resources. Under these circumstances, investment in repairers' health policies becomes absolutely necessary in order to ensure a minimum equity of healthcare systems in that state.
\end{abstract}

KEY W ORDS - Health Programs; National Diabetes Program; Chronic Care Self-Care Management.

\section{INTRODUCTION}

Neglected tropical diseases are a diverse group of tropical infections that are chronic in nature and especially common in low-income populations in developing regions of Africa, Asia, and the Americas. They are caused by a variety of pathogens such as viruses, bacteria, protozoa and helminths. Neglected tropical diseases have been in existence in Ghana for many years with many projects taking place to eradicate them but till now there are still places in Ghana still endemic to these diseases. The importance of neglected tropical diseases has been underestimated since many are asymptomatic and have long incubation periods. Areas of high endemicity are often in geographically isolated areas, making treatment and prevention much more difficult.

\section{Purpose of the study:}

The aim of the research is to the study cause of prevalence and endemicity of the main neglected tropical diseases in Ghana despite various attempts to eradicate them over the years and to proposed a plan to facilitate their eradication.
Article history: Submitted 29 March 2019 Accepted 19 June 2019

\section{Material and Method:}

Analysis of data from World Health organization (annual report 2000-2017), Ministry of health Ghana annual report 1998-2017, Ghana neglected tropical diseases program (5-year strategic plan -2013-2017), scientific articles, expert analysis, statistical methods. Examined the conditions, the category of the people affected, primary health care systems, the nature of the disease in endemic communities etc.

\section{RESULTS}

Analysis index of main neglected tropical diseases in Ghana which include the following:

a)Soil transmitted helminthiasis from 2012, number of cases 540,733 representing $(2,1 \%)$ of infected population, 2013: number of cases 804,472 (2.58\%), 2014: 855,677 (3.1\%), 2015: 830,557 (3.1\%), 2016: 816,106 (3.1\%). Major Soil-transmitted Helminths in Ghana are Ascaris lumbricoides, Trichuris trichuria, Necator americanus which causes malnutrition, anemia, growth retardation, cognitive impairment as well as lowering of resistance to other infections.

b) Lymphatic Filariasis Lymphatic Filariasis is currently was endemic in 98 out of the 216 districts in 8 regions of Ghana aside Ashanti and Volta regions in 2014 but currently 15 districts out of the 98 endemic districts remain endemic is still undergoing mass drug administration.

(c) Onchocerciasis: Remapping for onchocerciasis in 2009 using the rapid epidemiological mapping for oncho (REMO) methodology. The REMO results indicated 29 districts were hyperendemic (nodule prevalence $\geq 60 \%$ ), 15 districts were meso endemic (nodule prevalence 40-59.9\%), 91 districts were hypo endemic (nodule prevalence $<40 \%$ ) and the remaining 81 districts were non-endemic (nodule prevalence 0\%) In Ghana currently Onchocerciasis has an estimated at-risk population of 4,7 million in 3115 communities in 85 endemic districts from nine out of the ten regions and a possibility of increase to 125 endemic districts.

(d) Schistosomiasis: Urinary Schistosomiasis is also known as Bilharzia, caused by a blood fluke Schistosoma haematobium.In 2007, it was widespread and found in all regions in the Country, 47 category A 
(prevalence $\geq 50 \%$, high-risk) districts, 138 category $\mathrm{B}$ (prevalence $\geq 10-49.9 \%$, moderate risk) districts and 31 category C (prevalence 1-9.9\%, low-risk) districts. Results of the impact assessment indicated significant improvement in SCH in Ghana. Category A endemic districts reduced from 47 to 3 while category $\mathrm{B}$ reduced from 138 to 54 . The results were reviewed by an expert meeting in August 2016. Out of currently 254 districts in Ghana, $83 \%$ proportion of the districts are endemic for Schistosomiasis. (GHS 2016)

\section{CONCLUSION}

Despite the various projects and attempts made by the government and international organizations to eradicate neglected tropical diseases, We found out there is still much to be done in terms of prevention of the diseases in creating awareness and educating the public on the causes and effects of these diseases. 9 out of the 14 neglected diseases are still prevalent in Ghana of which 5 are endemic in most rural communities. Access to health care in the rural areas is very necessary in eradicating these diseases. Prevention and eradication are important because "of the appalling stigma, disfigurement, blindness and disabilities caused by NTDs. Research is still in progress.

\section{REFERENCES}

1. Hotez PJ, Kamath A (2009). Cappello, MichaEL, ED. Neglected Tropical Diseases in Sub-Saharan Africa 2. World Health Organization. Geneva: WHO; 2013. Lymphatic Filariasis: Fact sheet No 10. [Google Scholar]

3. End Neglected tropical diseases in Africa, Annual work plan Oct.2017-Sept 2018.https://endinafrica. org/wp-content/uploads/2018/04/Ghana-FY18Workplan-Final.pdf 